\title{
INCLUDING IONISATION IN A SIMPLE MODEL OF SINGLE-BUBBLE SONOLUMINESCENCE
}

\author{
ANGUS I. S. MUNRO ${ }^{1}$ and LARRY K. FORBES ${ }^{M} 1$
}

(Received 10 September, 2004; revised 7 January, 2006)

\begin{abstract}
A small gas bubble in a liquid, when driven by intense ultrasound, collapses and emits light in a process called Single-Bubble Sonoluminescence (SBSL). While the dynamics of driven bubbles are well studied, less is known of the physical conditions in the gas or whether it is necessary to include ionisation in simpler studies of bubble dynamics. In this study, a model was derived from Rayleigh-Plesset dynamics, a van der Waals equation of state and the first law of thermodynamics (including interfacial heat transfer and ionisation). Stronger model ionisation reduced the maximum collapse temperature, and altered other collapse characteristics. Chaotic parameter regions are proximal to, but not coincident with, known stable SL regions. Resonant behaviour was only markedly affected by ionisation close to these chaotic regions.
\end{abstract}

2000 Mathematics subject classification: 37N10, 76N99, 80A99, 65L07.

Keywords and phrases: buble dynamics, sonoluminescence, thermodynamics, ionisation, bifurcation, resonance maps, chaos.

\section{Introduction}

Sonoluminescence (SL) is a phenomenon whereby light is emitted from collapsing gas bubbles in a liquid. The high gas temperature required for SL is produced by quasi-adiabatic bubble collapse, facilitated by acoustic driving.

SL occurs in two forms: Single-Bubble (SBSL), and Multi-Bubble (MBSL). This study concerns SBSL, which was first observed in 1989 [7]. SBSL is easier to model than MBSL, because radial symmetry can be assumed under appropriate conditions. The SBSL system is a nonlinear oscillator, forced by an acoustic pressure field (typically ultrasound). This forcing induces cycles of cavitating expansion followed by collapses.

\footnotetext{
'School of Mathematics and Physics, University of Tasmania, Private Bag 37, Hobart, Tasmania, Australia; e-mail: Larry.Forbes@utas.edu.au.

(C) Australian Mathematical Society 2006, Serial-fee code 1446-1811/06
} 
Bubble collapse usually occurs at least once each cycle. The bubble, after being forced to expand to many times its normal radius, accelerates inwards under the increasing-pressure phase of driving. This leads to a bounce, at which instant the gas molecules are hard-packed, temperature reaches the order of $10^{4} \mathrm{~K}$ and light may be emitted. A more comprehensive summary of SBSL is given in the review by Brenner et al. [2].

Areas of application of bubble dynamics include medicine, materials science, chemistry, physical electronics, plasma physics, engineering, and marine biology [2]. For example, high pressures and temperatures near collapsing bubbles allow chemical reactions that may not otherwise occur. The interaction of microbubbles with ultrasound may enhance drug delivery in human blood [39]. Microbubble-induced mechanical or free radical action within tissue has been under study as a non-invasive treatment for cancer [38].

Early bubble oscillator models made many approximations (such as a polytropic, uniform, equilibrated gas) which were simple enough to allow numerical resonance studies [22]. More recent models incorporate vapour transport, chemical reactions, ionisation, radiation and even full gas hydrodynamics. Such models are described in [2]. Despite recent increases in available computing power, these sophisticated models are still prohibitively cumbersome for computational studies of bubble resonance and bifurcation. Resonance studies still seem restricted to simplified models, such as those of Lauterborn et al. [22, 24], Parlitz et al. [31] and Simon et al. [36]. Thus little is known of the effect of processes such as gas ionisation on bubble resonant behaviour.

This study presents a relatively simple model of a spherical bubble, which is, however, extended to include gas ionisation. The model includes a first-order chemical scheme for ionisation, within a simple model based on Rayleigh-Plesset dynamics and a uniform gas. Thermal behaviour will be derived directly from the first law of thermodynamics, giving a temperature parametrisation avoiding the assumptions of a polytropic law. A similar use of the first law has recently been described by Harris et al. [11]. The present model is still simple enough to investigate the parametric dependence of resonance.

Once the new model is developed, the effect of different ionisation strengths on bubble motion will be investigated. This will involve observation of the model bubble's collapse and resonance characteristics, while paying attention to the stability regime in which such a simple model is valid. It will be shown that including ionisation leads to cooler, longer collapses with slightly less time between afterbounces.

Spherical symmetry will be assumed in the model. Shape instabilities (which lead to departure from this symmetry) are believed to restrict the model's validity to bubbles of equilibrium radii between approximately $3 \mu \mathrm{m}$ and $5 \mu \mathrm{m}$ [15], frequencies below approximately $35 \mathrm{kHz}$ and driving below approximately $1.45 \mathrm{~atm}$ [12]. This stability 
region is not well defined; different physical conditions are suspected to extend the parameter range of stability [16]. Stable, non-spherical oscillations have produced SL outside this region $[7,19,25]$. The parameters used for this study are mostly within this region. Sometimes larger radii were used, and it remains unclear whether such conditions lead to stable bubbles.

While an undriven bubble will slowly dissolve due to the excess pressure created by surface tension, driven bubble systems which satisfy certain conditions (such as high dissolved gas content in the liquid) will increase in mass and size over many cycles by a process called rectified diffusion [2]. Such bubbles are unstable and this model will not apply. Because gas diffusion is ignored in this model, we mention that diffusive stability affects the region of validity of the model's results but not how these results are affected. For a detailed discussion, see the review of Brenner $e t$ al. [2].

Driven nonlinear oscillators, such as this bubble model, exhibit elaborate resonance structures. Using the notation of Lauterborn, Parlitz and co-workers [22,32], a nonlinear resonance is denoted $R_{n, m}$, where $n$ is its torsion number and $m$ is its period. Resonance behaviour will be compared with the results of previous studies [24, 31, 36]. In particular, it will be shown here that the chaotic parameter region is little affected by ionisation, and moves to larger bubbles for lower driving frequencies.

\section{Model}

2.1. Derivation Single-bubble systems have been under study since Lord Rayleigh quantified the spherical bubble in 1917 [34], in an equation modified in 1949 by Milton Plesset [33] and now known as the Rayleigh-Plesset Equation (RPE). The RPE takes the form

$$
R \ddot{R}+\frac{3}{2} \dot{R}^{2}=\frac{1}{\rho}\left(p_{g}-P_{0}-P(t)-4 \eta \frac{\dot{R}}{R}-\frac{2 \sigma}{R}\right) .
$$

Here, the fluid surrounding the bubble has density $\rho$, dynamic viscosity $\eta$ and surface tension $\sigma$ at the bubble wall. The ambient pressure in the fluid is $P_{0}$, and $P(t)$ is the pressure field of the applied acoustic forcing. The bubble's radius is $R(t)$, and the gas pressure within is denoted $p_{g}$. Values for constants are given in Table 1 .

The Rayleigh-Plesset Equation becomes inaccurate when the velocity of the bubble wall is comparable to the speed of sound in the liquid, $c$. The discrepancy stems from damping of the bubble's motion by its own sound emission, as reviewed in [2]. To account for this, various modifications to the RPE have been used. The version used for this study is

$$
\rho\left(R \ddot{R}+\frac{3}{2} \dot{R}^{2}\right)=\left[p_{g}-P_{0}-P(t)\right]-4 \eta \frac{\dot{R}}{R}-\frac{2 \sigma}{R}+\frac{R}{c} \dot{p}_{g},
$$


TABLE 1. Notation and values [26] of constants

\begin{tabular}{llll}
\hline Density (water) & $\rho$ & 1000 & $\mathrm{~kg} \mathrm{~m}^{-3}$ \\
Dynamic viscosity (water) & $\eta$ & $8 \times 10^{-4}$ & $\mathrm{~kg} \mathrm{~m}^{-1} \mathrm{~s}^{-1}$ \\
Surface tension (water) & $\sigma$ & $7.28 \times 10^{-2}$ & $\mathrm{~N} \mathrm{~m}^{-1}$ \\
Speed of sound (water) & $c$ & 1483.2 & $\mathrm{~m} \mathrm{~s}^{-1}$ \\
Universal gas constant & $\mathfrak{N}$ & 8.314 & $\mathrm{~J} \mathrm{~K}^{-1} \mathrm{~mol}^{-1}$ \\
van der Waals (air) & $\mathrm{A}$ & 0.1391 & $\mathrm{~Pa} \mathrm{~m}^{6} \mathrm{~mol}^{-2}$ \\
van der Waals (air) & $\mathrm{B}$ & $3.72 \times 10^{-5}$ & $\mathrm{~m}^{3} \mathrm{~mol}^{-1}$ \\
Interface heat transfer coefficient (estimated) & $c_{b}$ & $10^{3}$ & $\mathrm{~W} \mathrm{~m}^{-2} \mathrm{~K}^{-1}$ \\
Specific heat capacity (air) & $c_{v b}$ & 717.8 & $\mathrm{~J} \mathrm{~K}^{-1} \mathrm{~kg}^{-1}$ \\
\hline
\end{tabular}

which incorporates a sound-radiation term of order $\dot{R} / c$ times the other terms $[18,28]$. Solutions to (2.1) differ considerably from solutions to the RPE at bubble collapse, and are much more accurate [27].

The ability of uniform-gas interior bubble models to reproduce SBSL bubble dynamics has been a subject of debate. Undoubtedly such models cannot capture the finest features of bubble collapse and luminescence. Early theories proposed shock waves in the gas as the cause of SBSL; however, since then, mounting evidence suggests otherwise $[4,41]$. There is evidence that the collapsing bubble develops a boundary layer of water vapour and chemical reaction species, in what is termed mass segregation [37]. Such effects are not captured in uniform gas models; their significance in determining overall bubble dynamics is uncertain. Also, the involvement of such processes in luminescence does not imply that they impact bubble dynamics or overall collapse characteristics greatly, as the energies involved in luminescence are very small. We present a model more detailed than those in similar previous explorations of the parameter space, but still with a uniform bubble interior. As well as permitting us to explore large regions of parameter space, this permits some algebraic analysis of the system (both of which are impossible for most nonuniform-gas models due to their complexity). Having acknowledged the oversimplifications of this approach, we point out that research of Vuong and Szeri [41], Hilgenfeldt $e t$ al. [16] and Moss et al. [29] nevertheless suggests that uniform gas models are reasonably accurate.

We cast the bubble's uniform gas pressure term, $p_{g}$, in terms of bubble volume and gas temperature, through an equation of state (EOS). Because very high pressures are achieved, it is necessary to use the van der Waals equation [1],

$$
\left(p_{g}+A(n / V)^{2}\right)(V-n B)=n \Re T,
$$

where molar amount, volume and temperature are represented by $n, V$ and $T$ respectively. Constants $A$ and $B$ represent molecular interaction strength and molar 
hard-packed gas volume respectively.

It has been established indirectly that, over time, most of the gas in SL bubbles reacts and dissolves into the water [28], leaving the noble gas content (for example, Argon). While this is the case for the high temperatures in SL, the present model was designed to model bubble dynamical behaviour over a larger parameter space, in which rectification does not always take place. Because of the unknowns in this aspect, we have used atomic weight, heat capacity and van der Waals constants for air. This may be a source of error in regions where noble gas rectification occurs.

The time-dependent forcing pressure is

$$
P(t)=P_{\infty 0} \alpha \sin (2 \pi f t) .
$$

When (2.2) and (2.3) are substituted into the modified RPE, Equation (2.1), the equation of motion,

$$
\begin{aligned}
\ddot{R}= & \frac{1}{\rho R}\left\{\left(\frac{T}{k_{0} R^{3}-k_{1}}-\frac{k_{2}}{R^{6}}-P_{\infty 0}(1+\alpha \sin (\omega t))\right)-4 \eta \frac{\dot{R}}{R}-\frac{2 \sigma}{R}\right\} \\
& +\frac{1}{\rho c}\left(\frac{\dot{T}}{k_{0} R^{3}-k_{1}}-\frac{3 k_{0} T \dot{R} R^{2}}{\left(k_{0} R^{3}-k_{1}\right)^{2}}+\frac{6 k_{2} \dot{R}}{R^{7}}\right)-\frac{3}{2} \frac{\dot{R}^{2}}{R},
\end{aligned}
$$

is obtained, where $k_{0}=4 \pi /(3 n 9), k_{1}=B / 9$ and $k_{2}=9 A n^{2} /\left(16 \pi^{2}\right)$.

Both the bubble radius $R(T)$ and the gas temperature $T(t)$ are unknown in (2.4) and therefore a consideration of thermodynamics is needed to close the system. This is discussed below.

Many uniform-gas bubble models incorporate heat transfer indirectly through the use of a polytropic exponent $[7,13,14,16,36]$, in which gas pressure and density within the bubble are related by a simple algebraic equation. Close to collapse, the bubble is almost adiabatic [2], while interfacial heat transfer is significant at other times. Some models use discrete polytropic schemes in different parts of the cycle to cope with this, which makes algebraic analysis very difficult. Many detailed models additionally include the thermodynamics of chemical processes occurring in the bubble and interface. For simplicity these are not done here; interfacial heat transfer is treated as Newtonian, while chemical reactions and ionisation are treated in one broad mathematical swoop. This is not expected to provide all the answers to what happens at bubble collapse, but it will hopefully reflect the overall dynamical qualities of the collapsing, reacting, ionising bubble system reasonably.

In this model, the van der Waals EOS is used in conjunction with the First Law of Thermodynamics. The First Law states that any change in internal energy of a volume of gas equals the sum of work done and heat input. Integrated over the entire bubble, this may be written

$$
\frac{d E_{b}}{d t}+p_{b} \frac{d V_{b}}{d t}=\dot{Q}_{h}
$$


where $E_{b}$ is the total internal energy and $\dot{Q}_{h}$ is the total rate of surface heat transfer into the bubble. Expressing volume in terms of radius and internal energy in terms of temperature gives

$$
M_{b} c_{v b} \frac{d T}{d t}+4 \pi R^{2} \frac{d R}{d t} p_{b}=\dot{Q}_{h},
$$

where $c_{v b}$ is the specific heat of gas at constant volume and $M_{b}$ is bubble mass (derived from the stipulated bubble rest radius at 1 atm ambient pressure). In this model, the net heat transfer from the bubble is written

$$
\dot{Q}_{h}=\dot{Q}_{c}+\dot{Q}_{i}
$$

where subscripts $c$ and $i$ represent bubble wall heat conduction and gas ionisation respectively.

The first term in (2.6) is the interfacial conductive heat transfer rate, $\dot{Q}_{c}$. This depends on the bubble's surface area and the temperature difference across the interface, and is given by Newton's law of cooling in the form

$$
\dot{Q}_{c}=4 \pi R^{2} c_{b}\left(T_{w}-T\right),
$$

where $c_{b}$ is a constant of proportionality and $T_{w}$ is water temperature.

Additional thermodynamic effects, such as chemical reactions and ionisation, are represented by a single lumped ionisation reaction,

$$
A \rightleftharpoons A^{+}+e^{-},
$$

in which the reaction rates are functions of temperature. This reaction serves to store system energy at times of high bubble temperature. Thermodynamic equilibrium is assumed [20]. The scheme is written as a single ionisation, but this may be considered to parametrise the energy absorbed by single ionisation, multiple ionisations and chemical reactions. The fraction, $\iota$, of gas "ionised" in this way is calculated on the basis of Arrhenius kinetics to have the form

$$
\iota(T)=e^{-\zeta / T} .
$$

From the bubble's total energy of ionisation, $-Q_{i}=n I \iota(T)$, the ionisation rate immediately follows,

$$
\dot{Q}_{i}=-\zeta n I e^{-\zeta / T} \dot{T} / T^{2},
$$

which is the second term in (2.6). The effect of this model component is to absorb heat energy at high temperatures - thereby mitigating peaks in temperature - and releasing the heat energy again as the temperature falls. 
Equation (2.6) thus shows that the total rate of heat input into the bubble is

$$
\dot{Q}_{h}=4 \pi R^{2} c_{b}\left(T_{w}-T\right)+\zeta n I e^{-\zeta / T} \dot{T} / T^{2} .
$$

Substituting the heat transfer rate (2.7) into (2.5) and rearranging gives

$$
\dot{T}=\frac{\left(4 \pi R^{2} / M_{b}\right)\left[c_{b}\left(T_{w}-T\right)-\dot{R}\left(T /\left(k_{0} R^{3}-k_{1}\right)-k_{2} / R^{6}\right)\right]}{c_{v b}+\zeta\left(I / m_{m}\right) e^{-\zeta / T} / T^{2}} .
$$

The molar mass of gas is $m_{m}$.

The forced, highly nonlinear system of ordinary differential equations, (2.4)-(2.8), describes the behaviour of the forced single-bubble system.

In the remainder of this paper, a non-dimensional form of these equations is used. All quantities are scaled by:

$$
\begin{aligned}
\text { length scale: } & L_{s}=2 \sigma / p_{\infty 0} \\
\text { time scale: } & \tau_{s}=\left(2 \sigma / p_{\infty 0}\right) \sqrt{\left(\rho / p_{\infty 0}\right)} \text { and } \\
\text { temperature scale: } & T_{s}=\zeta .
\end{aligned}
$$

Note that $\rho$ is water (not air) density, so the velocity scale does not relate to the speed of sound.

In nondimensional form, the system becomes

$$
\begin{aligned}
\frac{d R}{d t}= & v, \\
\frac{d v}{d t}= & \frac{1}{R}\left\{\xi\left(\frac{T}{R^{3}-H^{3}}-\frac{\kappa_{1}}{R^{6}}\right)-\phi(1+\alpha \sin \omega t)-\frac{1}{R}(\lambda v+\beta)\right. \\
& \left.+\kappa_{2} \frac{v}{R^{6}}-\frac{3}{2} v^{2}\right\}+\frac{\psi}{R^{3}-H^{3}}\left(F-\frac{3 R^{2} v T}{R^{3}-H^{3}}\right), \\
\frac{d T}{d t}= & F, \\
F= & 4 \pi R^{2}\left\{\frac{\chi\left(\theta_{w}-T\right)-v\left(T /\left(R^{3}-H^{3}\right)-\kappa_{1} / R^{6}\right)}{\mu+\epsilon e^{-\delta / T} / T^{2}}\right\},
\end{aligned}
$$

with the dimensionless constants

$$
\begin{aligned}
\xi & =3 M_{b} \Re T_{s} \tau_{s}^{2} /\left(4 \pi m_{m} \rho L_{s}^{5}\right), & H^{3} & =3 M_{b} B /\left(4 \pi m_{m} L_{s}^{3}\right), \\
\kappa_{1} & =3 M_{b} A /\left(4 \pi m_{m} \Re L_{s}^{3} T_{s}\right), & \phi & =p_{\infty 0} \tau_{s}^{2} /\left(\rho L_{s}^{2}\right), \\
\omega & =2 \pi f \tau_{s}, & \lambda & =4 \eta \tau_{s} /\left(\rho L_{s}^{2}\right), \\
\beta & =2 \sigma \tau_{s}^{2} /\left(\rho L_{s}^{3}\right), & \psi & =3 \tau_{s} T_{s} M_{b} \Re /\left(4 \pi m_{m} c \rho L_{s}^{4}\right), \\
\kappa_{2} & =\left(3 M_{b} /\left(4 \pi m_{m}\right)\right)^{2} 6 A T_{s} /\left(\rho c L_{s}^{7}\right), & \chi & =4 \pi m_{m} c_{b} \tau_{s} L_{s}^{2} /\left(3 M_{b} \Re\right), \\
\mu & =4 \pi c_{v b} m_{m} /(3 \Re), & \theta_{w} & =T_{w} / T_{s}, \\
\epsilon & =4 \pi \zeta I /\left(3 \Re T_{s}^{2}\right) & \delta & =\zeta / T_{s} \text { and } \alpha .
\end{aligned}
$$


Although the model notionally involves 15 dimensionless parameters (each being dependent on several physical parameters), only four physical parameters can be meaningfully varied in an experimental situation. These are forcing frequency $(f)$, forcing amplitude $(\alpha)$ (specified as a fraction of the ambient liquid pressure), water temperature $\left(T_{w}\right)$ and ambient liquid pressure $\left(p_{\infty 0}\right)$. Bubble ambient radius, $\left(R_{e}\right)$, is controlled by these parameters; however, $\left(R_{e}\right)$ is often visualised as an additional independent variable in research on similar models $[3,7,31]$.

This study will chiefly investigate the dependence of model behaviour upon gas ionisation temperature $(\zeta)$ and gas ionisation energy $(I)$. These variables were chosen because they are important to this model's parametrisation of energy exchange (which is an extension of that of previous dynamical models). The forcing and liquid parameters mentioned in the previous paragraph will still be manipulated to produce maps, as has been done previously. However, the effect of the ionisation parameters on these maps and on bubble collapse behaviour will also be assessed.

2.2. Equilibria and small-amplitude oscillations In this section, some analytical procedures will be applied to the model (Equations (2.9) prior to their full numerical implementation). These will demonstrate physically relevant attributes such as solution stability and the characteristics of resonance.

Firstly, equilibrium points will be located. Then, by perturbing in a similar manner to Forbes [5], their stability, local behaviour and resonant frequency shall be found.

Equilibrium points Equilibrium points of the undriven $(\alpha=0)$ system are found by setting the three derivatives on the left-hand side of (2.9) to zero. From (2.9c), the equilibrium temperature must equal the ambient water temperature, $\theta_{w}$. The equilibrium bubble radius, $R_{\ell}$, is then determined from $(2.9 \mathrm{~b})$ to satisfy the algebraic equation

$$
\theta_{u}=\left(R_{e}^{3}-H^{3}\right)\left(\frac{\kappa_{1}}{R_{e}^{6}}+\frac{\phi}{\xi}+\frac{\beta}{\xi R_{e}}\right)
$$

A single equilibrium temperature, $T_{e}\left(=T_{w}\right.$ ), can be defined for each value of $R_{e}$. Equilibrium radii were plotted against $T_{w}$, as shown in Figure 1 . In the physically relevant parameter region for SBSL dynamics, there exists a single, stable solution.

Water temperature has been shown to strongly affect the amount of light emission in SBSL [40], but this is due to the effect of water vapour in the bubble. Water temperature changes complicate the system by affecting viscosity, reactions and other things; the study of such properties of the medium constitutes its own field of research. To keep matters simple, water temperature was kept at $293 \mathrm{~K}$ throughout this study.

This solution changed qualitatively for temperatures $\approx 100 \mathrm{~K}$, due to van der Waals gas behaviour. The behaviour of gas bubbles at these very low temperatures - 


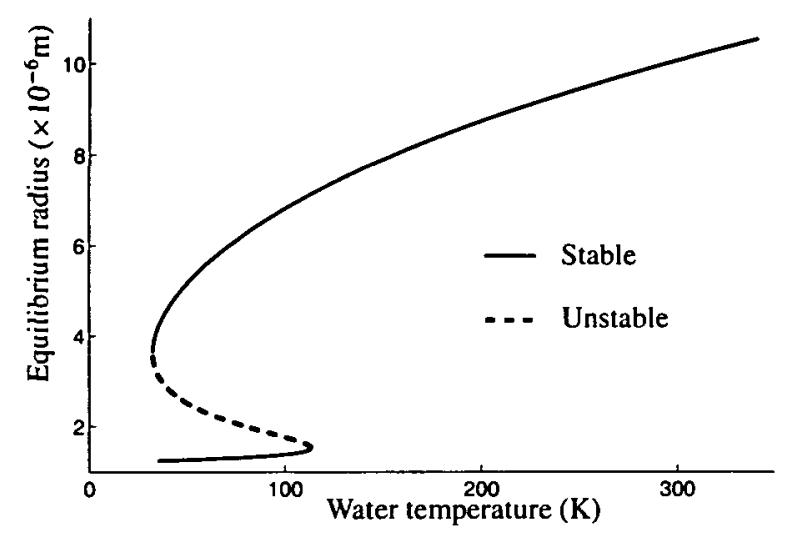

FIGURE 1. Relationship between equilibrium bubble radius $\left(R_{e}\right)$ and water temperature $\left(T_{w}\right)$.

requiring liquids other than water and different gases - would be an interesting topic for further study.

Local behaviour Once the system's equilibrium point(s) are found from (2.10), the behaviour of nearby trajectories is determined by linearisation and the HartmanGrobman theorem [8]. The linearised, unforced system can be derived from an expansion about equilibrium values, in the form

$$
\begin{aligned}
R(t) & =R_{e}+E R_{1}(t)+O\left(E^{2}\right), \\
v(t) & =0+E v_{1}(t)+O\left(E^{2}\right), \\
T(t) & =T_{e}+E T_{1}(t)+O\left(E^{2}\right),
\end{aligned}
$$

in which $E$ is a measure of some small departure from equilibrium. The first-order terms then satisfy the linearised matrix system

$$
\frac{d}{d t}\left(\begin{array}{c}
R_{1} \\
v_{1} \\
T_{1}
\end{array}\right)=J\left(\begin{array}{c}
R_{1} \\
v_{1} \\
T_{1}
\end{array}\right)
$$

where the matrix $J$ is the usual Jacobian matrix of partial derivatives,

$$
J=\left(\begin{array}{lll}
\partial f_{1} / \partial R & \partial f_{1} / \partial v & \partial f_{1} / \partial T \\
\partial f_{2} / \partial R & \partial f_{2} / \partial v & \partial f_{2} / \partial T \\
\partial f_{3} / \partial R & \partial f_{3} / \partial v & \partial f_{3} / \partial T
\end{array}\right)=\left(\begin{array}{lll}
j_{11} & j_{12} & j_{13} \\
j_{21} & j_{22} & j_{23} \\
j_{31} & j_{32} & j_{33}
\end{array}\right) .
$$

The evaluated elements of $J$ can be found in the Appendix.

Eigenvalues of the Jacobian matrix may be computed using MATLAB, and indicate the behaviour of the linearised solution. The equilibrium configuration is only stable if all these eigenvalues have negative real parts; see Guckenheimer and Holmes [8]. 
Small-amplitude forcing and resonances The next analytical step is to investigate the effect of slight acoustic forcing. Using the rationale of Forbes [6], the pressureforcing amplitude is assumed to be a function of radial perturbation amplitude. By including the driving pressure $P(t)=-\phi \alpha \sin \omega t(\alpha \ll 1)$ on the right-hand side of $(2.9 \mathrm{~b})$, the small-amplitude resonance behaviour of the system was derived. The system becomes

$$
\frac{d}{d t}\left(\begin{array}{c}
R_{1} \\
v_{1} \\
T_{1}
\end{array}\right)=J\left(\begin{array}{c}
R_{1} \\
v_{1} \\
T_{1}
\end{array}\right)+\left(\begin{array}{c}
0 \\
-\phi \\
0
\end{array}\right) \sin (\omega t)
$$

and its solution (particular integral) is of the form

$$
\begin{aligned}
& R_{1}(t)=M_{1} \cos (\omega t)+N_{1} \sin (\omega t), \\
& v_{1}(t)=\omega\left(N_{1} \cos (\omega t)-M_{1} \sin (\omega t)\right), \\
& T_{1}(t)=P_{1} \cos (\omega t)+Q_{1} \sin (\omega t),
\end{aligned}
$$

where (2.12a) was substituted into the first row of the matrix system (2.11) to give (2.12b). Equations (2.12) were then substituted into the two remaining equations in system (2.11), to give two identities in $\cos (\omega t)$ and $\sin (\omega t)$. These produced the following system of algebraic equations:

$$
\begin{aligned}
N_{1}\left(\omega^{2}+a\right)-j \omega M_{1}+c Q_{1}-\phi & =0, \\
M_{1}\left(\omega^{2}+a\right)+b \omega N_{1}+c P_{1} & =0, \\
\omega\left(P_{1}-d M_{1}\right)+e Q_{1} & =0, \\
\omega\left(Q_{1}-d N_{1}\right)-e P_{1} & =0,
\end{aligned}
$$

with $a=j_{21}, b=j_{22}, c=j_{23}, d=j_{32}$ and $e=j_{33}$ for convenience. The solution to this system is the set of coefficients in the particular integral for the small-amplitude forced system, as functions of forcing angular frequency $\omega$.

The amplitude of $R_{1}(t)$ is $A=\sqrt{M_{1}^{2}+N_{1}^{2}}$. In terms of the solutions of (2.13),

$$
A=A(\omega)=\frac{\phi\left(\omega^{2}+e^{2}\right)}{\sqrt{\left[\left(a+\omega^{2}\right)\left(\omega^{2}+e^{2}\right)+c d \omega^{2}\right]^{2}+\left[\omega b\left(\omega^{2}+e^{2}\right)-\omega c d e\right]^{2}}} .
$$

Resonance occurs when $d A / d \omega=0$ for $d^{2} A / d \omega^{2}<0$. The first condition gives either $\omega=0$ or

$$
\begin{aligned}
0= & 2 \eta^{4}+\eta^{3}\left(2 a^{2}+b^{2}+2 c d+6 e^{2}\right)+\eta^{2} 3 e^{2}\left(2 a^{2}+b^{2}+2\left(c d+e^{2}\right)\right) \\
& +\eta e^{2}\left(c^{2} d^{2}+3 b^{2} e^{2}+2 e^{4}+2 c d e(2 e-b)+2 a^{2}\left(c d+3 e^{2}\right)\right) \\
& +e^{4}\left((c d-b e)^{2}+2 a^{2}\left(c d+e^{2}\right)\right)
\end{aligned}
$$


where $\eta=\omega^{2}$. Solving numerically for a $10 \mu \mathrm{m}$ air bubble at $293 \mathrm{~K}$, with $I=$ $1.35 \times 10^{6} \mathrm{~J} \mathrm{~mol}^{-1}$ and $\zeta=20000 \mathrm{~K}$, gave four real $\eta$ solutions, two negative and two positive. The physically real solution is the larger positive root, a maximum with $d^{2} A / d \omega^{2}<0$. The smaller positive solution is a low-frequency amplitude minimum $\left(d^{2} A / d \omega^{2}>0\right)$, while the imaginary square roots of the negative solutions are nonphysical. The amplitude curve shape is illustrated in Figure 2.

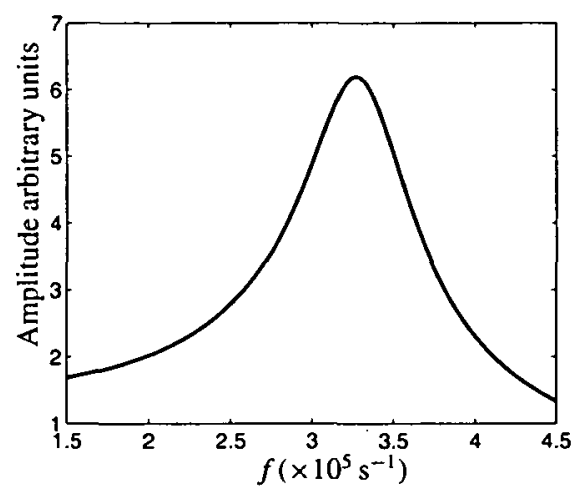

FIGURE 2. Frequency dependence of oscillation amplitude in the perturbed linearised system.

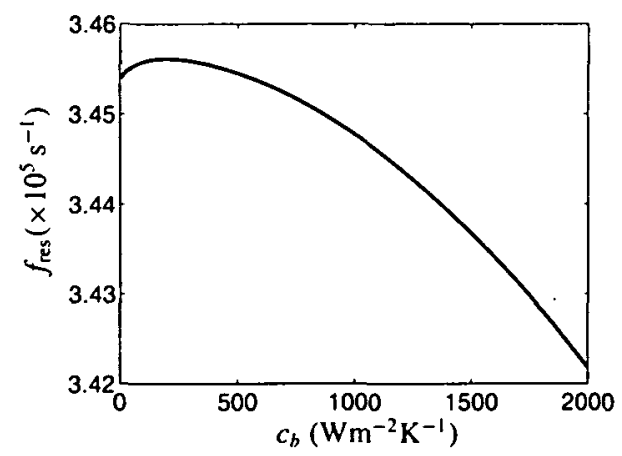

FIGURE 3. Effect of $c_{b}$ on resonant frequency of a $10 \mu \mathrm{m}$ bubble with normal ionisation.

The curve in Figure 2 peaks at a frequency, $f_{\text {res }}$, equal to the primary resonant frequency of the nonlinear system in the limit of small driving amplitude. This resonant frequency depends on most model parameters. Its dependence on $c_{b}$, the coefficient for heat transfer at the bubble wall, is shown in Figure 3. Here, the largest value of $f_{\text {res }}$ occurs when $c_{b} \sim 200 \mathrm{~W} \mathrm{~m}^{-2} \mathrm{~K}^{-1}$. The effect of the ionisation parameters $I$ and $\zeta$ on the resonant frequency was found to be very small. 
The damping in this system limits the oscillation amplitude at frequency $f_{\text {res }}$. An undamped oscillator would show an infinite peak at this frequency. The amount of damping in this system, and hence the peak height in Figure 2, approximately depends on element $j_{22}$ of the Jacobian matrix. The physical contributors to this matrix term, and therefore the system's damping, consist of liquid viscosity, gas thermodynamic parameters and ionisation parameters. The ionisation parameters are the least well known of these; knowledge of how sensitive the bubble motion is to errors in ionisation parameters would be useful for SBSL modelling. The remainder of this study will investigate this using a computer implementation of (2.9).

\section{Methods}

3.1. Numerical implementation To facilitate their solution in MATLAB, the nondimensionalised Equations (2.9) were transformed by the map

$$
x_{1}=h e^{u R / R_{e}}, \quad x_{2}=j v e^{u R / R_{e}}, \quad x_{3}=k \ln \left(w T / T_{e}\right),
$$

where $h, j, k, u$ and $w$ are constants. This is an extension of the map used by Parlitz et al. [31] to solve the RPE. Substituting (3.1) into (2.9), the system can be recast in terms of the derivatives of the new variables,

$$
\begin{aligned}
\frac{d x_{1}}{d t} & =x_{2} \frac{u h}{R_{e} j} \\
\frac{d x_{2}}{d t} & =\frac{j}{h}\left(\frac{d v}{d t}\right) x_{1}+x_{2} \frac{u v}{R_{e}}, \\
\frac{d x_{3}}{d t} & =\frac{k}{T}\left(\frac{d T}{d t}\right) .
\end{aligned}
$$

For simplicity, bracketed terms represent right-hand sides of the original Equations (2.9), and Equations (3.1) have not been fully substituted.

The transformation was used to minimise MATLAB's computational overhead (and error) by producing the set of variables $x_{1}, x_{2}$ and $x_{3}$ with smoother time-behaviour than $R, v$ and $T$. The inverse transformations are

$$
R=\left(R_{e} / u\right) \ln \left(x_{1} / h\right), \quad v=(h / j)\left(x_{2} / x_{1}\right), \quad T=\left(T_{e} / w\right) e^{x_{3} / k} .
$$

Equations (3.2) were integrated in MATLAB using the stiff equation solver ODE23S. The solution was then inverted using (3.3) to give $R, v$ and $T$.

Most stable resonances were reproduced almost identically by both the transformed and non-transformed methods. Bifurcation structures were essentially the same. Numerical noise in bifurcation diagrams was largely removed by the transformed 
solution method. The transformed solution method was used for the remainder of the study.

To produce bifurcation diagrams for this study, each cycle's maximum radius was recorded [36]. This method was used in preference to stroboscopic (regular) sampling because the latter displayed noise from transients. At each of $\sim 800$ horizontal parameter increments, the system was integrated over $\sim 150$ driving cycles. The first 50 cycles were discarded and the maximum radii of the remainder were plotted. The system was not restarted after each increment, to minimise transients associated with the change.

3.2. Ionisation parameters Following the derivation and implementation of the model, the main aim of this study was to investigate the model's collapse and resonant behaviours under different gas ionisation conditions.

This model's two adjustable ionisation parameters are $I$ and $\zeta$. These affect how the bubble absorbs heat energy in ionisation (and other chemical) processes. The parameter $I$ is the molar energy absorbed by these processes. The parameter $\zeta$ is a characteristic temperature at which these processes act. Such processes are most pronounced at high temperatures of several thousand $K$. Below is a brief description of how these two parameters were varied in this study.

Some of the investigations only compared two cases: "with" ionisation and "without". The default values used for the former case were calculated with the aim of being physically sensible. These values are $I=1.35 \times 10^{6} \mathrm{~J} \mathrm{~mol}^{-1}$ (which assumes a single ionisation at a level of $\sim 14 \mathrm{eV}$ per molecule), and $\zeta=20000 \mathrm{~K}$. These values will be hence referred to as "normal" ionisation.

The $\zeta$ value in normal ionisation translates to the gas being $\sim 13.5 \%$ ionised at $10000 \mathrm{~K}$ and $\sim 37 \%$ ionised at $20000 \mathrm{~K}$. This corresponds to slightly more ionisation (and, hence, more energy involvement) at a given temperature threshold than SL research suggests $[2,9]$. However, other effects such as chemical reactions (which, at bubble collapse, absorb heat energy in a similar way to ionisation) have not been included in this model, so a conservative $\zeta$ estimate was used to accommodate them. We emphasise that current knowledge of the energies involved in ionisation for SL is limited, so the values used here are estimates.

The effects of these parameters on the characteristics of both bubble collapse and resonance were examined, as summarised below.

3.3. Collapse characteristics A representative "cycle" was generated for a $10 \mu \mathrm{m}$ bubble driven at $26.5 \mathrm{kHz}$ and $1.6 \mathrm{~atm}$ (a period-1 oscillation close to the edge of the chaotic zone). This was first attempted by "winding up" the oscillator until a stable cycle was reached. However, this method was abandoned because it was extremely numerically sensitive, resulting in noise and poor repeatability. A simpler and more 
repeatable result was obtained by providing a typical pre-collapse initial condition (taken from a sample cycle), and then integrating the system for 1 driving cycle. The shape of the radius-time curve for 1 driving cycle was compared for a bubble with varying levels of ionisation and zero ionisation. Afterbounces were also examined, because these indicate the oscillator's cycle-to-cycle stability.

Next, the bubble's initial collapse was integrated, and properties of the collapse were recorded. These include: the full-width half-maximum $\left(\mathrm{FWHM}_{T}\right)$ of the temperature pulse, the maximum temperature $\left(T_{\max }\right)$, the maximum speed of the infalling bubble wall $\left(v_{\max }\right)$, and the total energy of ionisation $\left(E_{l}\right)$. This simulation was repeated at a grid of $50 \times 50$ combinations of $I$ and $\zeta$.

Collapse characteristics were compared with results from other published models, for example, Yasui [43], Ho [17] and Hilgenfeldt [14]. Most of these models are much more complicated than the system used here. They include processes such as gas hydrodynamics, detailed chemical reactions, vapour exchange and ionisation.

3.4. Resonance characteristics We next investigated the effect of ionisation on model behaviour over many cycles. While a series of bifurcation diagrams is useful to visualise the effect of two parameters on bifurcations, a more condensed method is to use 2-D maps which indicate the parameter areas of different resonances. These "resonance maps" are essentially collapsed bifurcation diagrams with an additional control parameter dimension. They can either show lines of bifurcation, or (equivalently) have image pixels shaded different colours for different resonances. The latter method was chosen for this study. The model was run over thousands of driving cycles at each combination of $I$ and $\zeta$ in a fine grid of pixels. Different periods of motion were allocated different colours, and a 2-D map was produced.

With a similar technique, maps in $R_{e}$ (initial bubble radius) and $\alpha$ (driving amplitude) were produced. Similar maps have been produced for a simpler bubble model [36]. In this study, maps were produced with and without ionisation to see if the location of the chaotic region was affected.

These maps have not been extensively used in the past, possibly because of the large computing resources required to produce them. They were used by Parlitz [30], Lauterborn and Mettin [23] and Simon et al. [36] to visualise the shape of resonance horns.

\section{Results and discussion}

As an introduction to this section, a typical oscillation cycle of the model is discussed. This is followed by a brief overview of the model's bifurcation structure. Results are then presented showing the effect of ionisation on the bubble's behaviour. 
4.1. Model behaviour The acoustic driving frequency $(\sim 20 \mathrm{kHz})$ is much lower than the bubble's natural oscillation frequency $(\sim 500 \mathrm{kHz})$. While small driving pressures produce bubble oscillations that are nearly linear, larger pressures of $\sim 1$ atmosphere produce violent, rebounding collapses.

Figure 4 displays two typical cycles. The bubble is under tension initially, producing a large expansion to over ten times its ambient radius. As pressure increases, this expansion halts and the bubble wall accelerates inwards. The infalling bubble wall, having begun its contraction at a large radius, achieves a very large velocity under the high acoustic pressure. The bubble wall continues to accelerate inwards until a bounce occurs, accompanied by a sharp temperature peak of approximately $10000 \mathrm{~K}$. This occurs as the bubble contents approach their van der Waals hard-core radius.

Following the initial collapse and rebound are several smaller "afterbounce" collapses. Eventually these dampen into steady oscillations close to the bubble's natural oscillation frequency. If the forcing frequency is low enough and damping mechanisms are sufficient, the afterbounces virtually disappear by the next cycle.

(a)
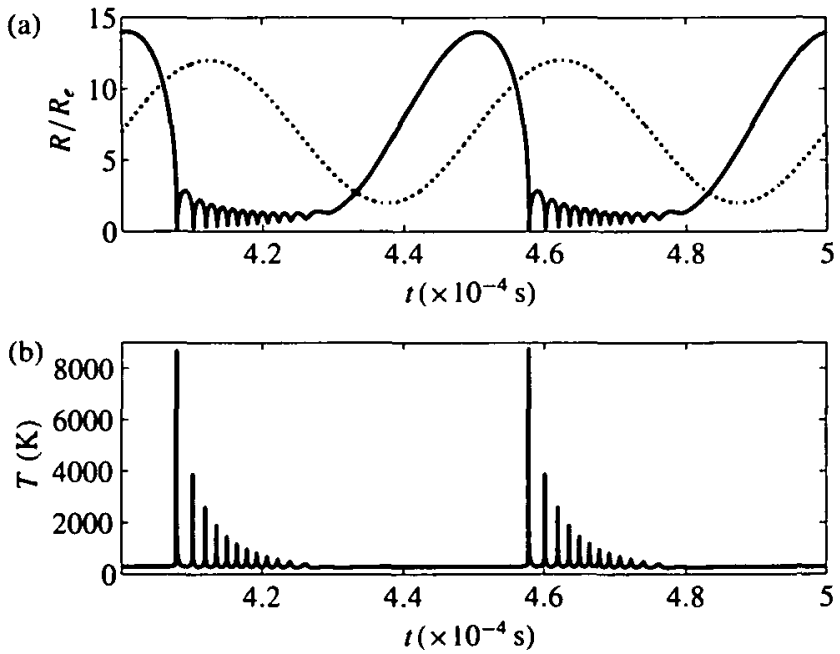

FIGURE 4. Typical oscillations of a $7 \mu \mathrm{m} \mathrm{SL}$ (ionising) air bubble driven by $1.6 \mathrm{~atm}$ ultrasound at $20 \mathrm{kHz}$ : (a) bubble radius, and (b) bubble temperature. The dotted line in (a) indicates the phase of acoustic driving.

Figure 5 shows a series of frequency bifurcation diagrams for a $10 \mu \mathrm{m}$ bubble. It is a useful illustration of the effects of forcing frequency $(f)$ and forcing amplitude $(\alpha)$ on bubble resonance. For small $\alpha$, the period-1 resonances $\left(R_{n .1}\right)$ dominate, and become more prominent as $\alpha$ is increased. Period-2 resonances $\left(R_{n .2}\right)$ are born suddenly in a period-doubling bifurcation as $\alpha$ is increased. This happens first at high 
frequencies, then for low frequencies at larger forcing amplitudes. Further increase of amplitude $\alpha$ increases the depth of bifurcation, and eventually chaos is reached. In Figure 5 (a), $\alpha$ is just large enough for the 2 subharmonics $R_{5,2}$ and $R_{7,2}$ to be present. In Figure 5 (b), $R_{5,2}$ has bifurcated to chaos, while $R_{7,2}$ has bifurcated at least to period $4\left(R_{14,4}\right)$. For $\alpha \gtrsim 0.9$, large regions of chaos are present; a period-3 window can be seen beneath $R_{3,1}$ in Figure 5 (d). This bifurcation structure is typical of those observed in nonlinear oscillators. For a more comprehensive description of bifurcations in bubble oscillators, refer to Parlitz et al. [31], Scheffczyk et al. [35] and Parlitz [30].

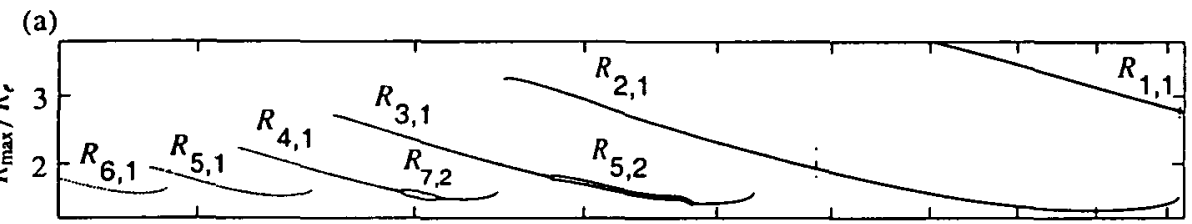

(b)

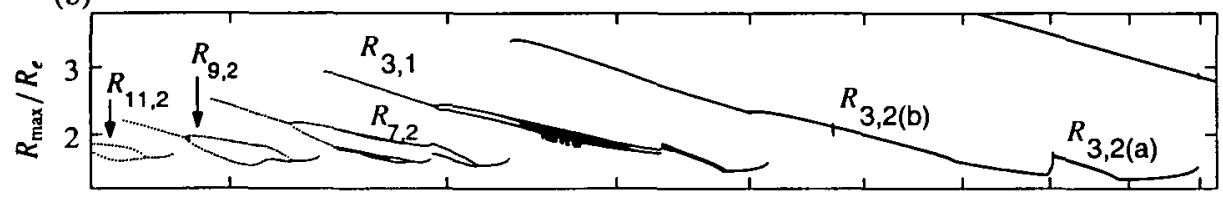

(c)

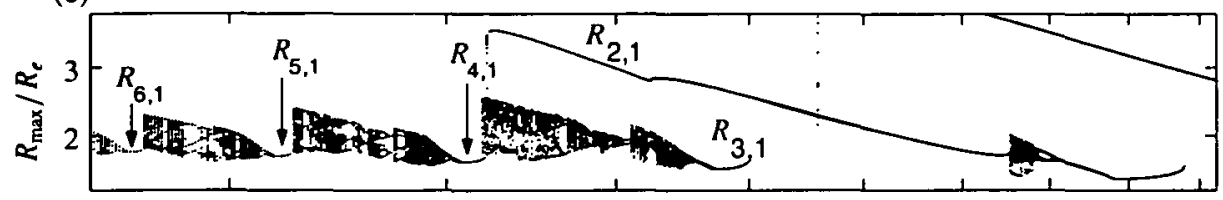

(d)

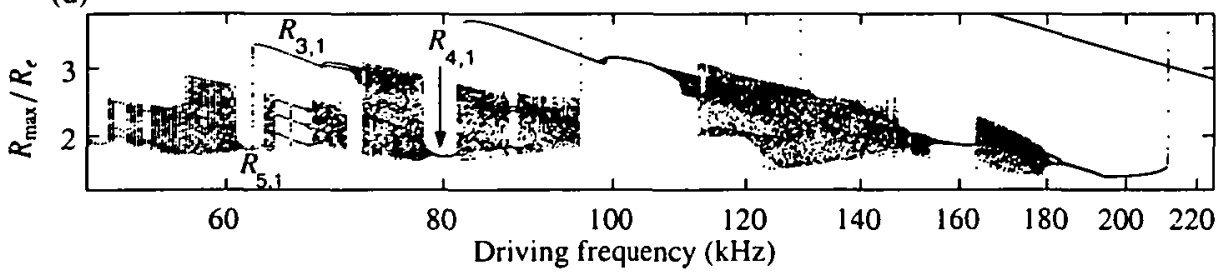

FIGURE 5. Sequence of frequency bifurcation diagrams, for (a) $\alpha=0.8$, (b) $\alpha=0.85$, (c) $\alpha=0.9$ and (d) $\alpha=0.95$ for a $10 \mu \mathrm{m}$, non-ionising bubble. Selected resonances are labelled.

4.2. Effects of ionisation on cycle and collapse characteristics Differences in radius-time behaviour were found between bubbles with $\zeta=10000 \mathrm{~K}$ and bubbles with $\zeta=20000 \mathrm{~K}$, as shown in Figure 6. Smaller, higher frequency afterbounces were produced for $\zeta=10000 \mathrm{~K}$. For $\zeta=10000 \mathrm{~K}, l$ had to be reduced by a factor of around 20 before the $R(t)$ curve resembled the $\zeta=20000 \mathrm{~K}$ (dashed) curve. Small 
differences in radius-time behaviour have been shown to alter bubble resonance when rebound oscillations are not damped away by the next cycle [36].

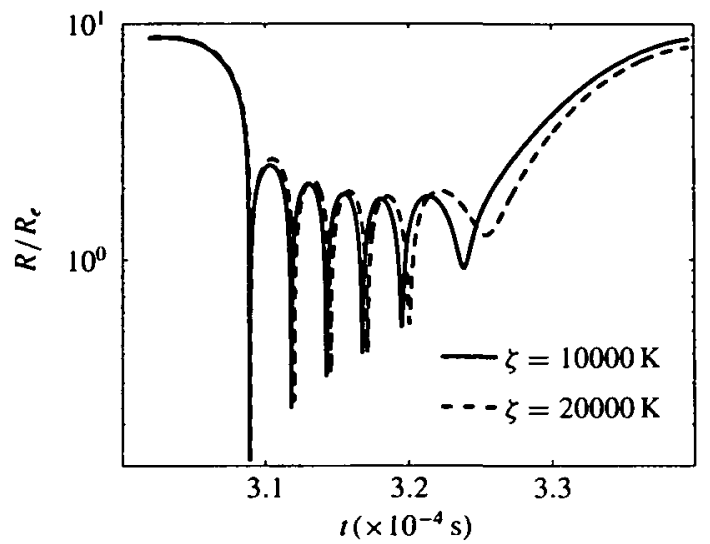

FIGURE 6. Effect of $\zeta$ on a $10 \mu \mathrm{m}$ bubble oscillation cycle, with $f=26.5 \mathrm{kHz}, \alpha=1.6$ and $I=$ $1.35 \times 10^{6} \mathrm{Jmol}^{-1}$

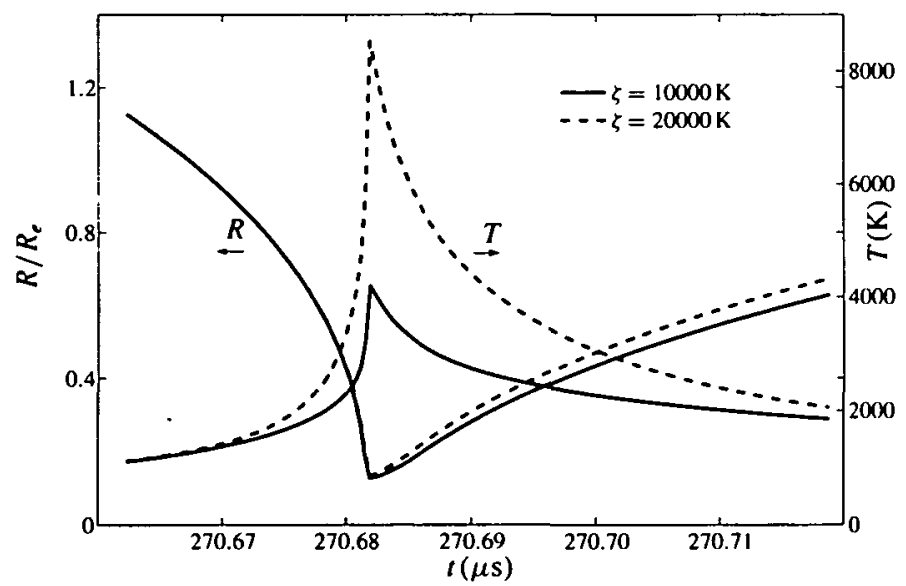

FiguRE 7. Effect of $\zeta$ on time-behaviour of a $10 \mu \mathrm{m}$ bubble collapse, with $f=26.5 \mathrm{kHz}, \alpha=1.6$ and $I=1.35 \times 10^{6} \mathrm{Jmol}^{-1}$.

A close-up of the collapse region is shown in Figure 7. Reducing $\zeta$ from $20000 \mathrm{~K}$ to $10000 \mathrm{~K}$ (both physically feasible values) approximately halved the bubble's maximum collapse temperature.

The minimum collapse radius was very similar for both ionisation scenarios, and was close to the van der Waals hard core radius. While not illustrated here, the minimum radius was smallest for large ionisations and largest with no ionisation. 
The more detailed investigation of extrema in bubble collapse properties involved contouring these extrema in the $I-\zeta$ plane, as shown in Figure 8. The same initial condition was used to generate all the data for these plots.
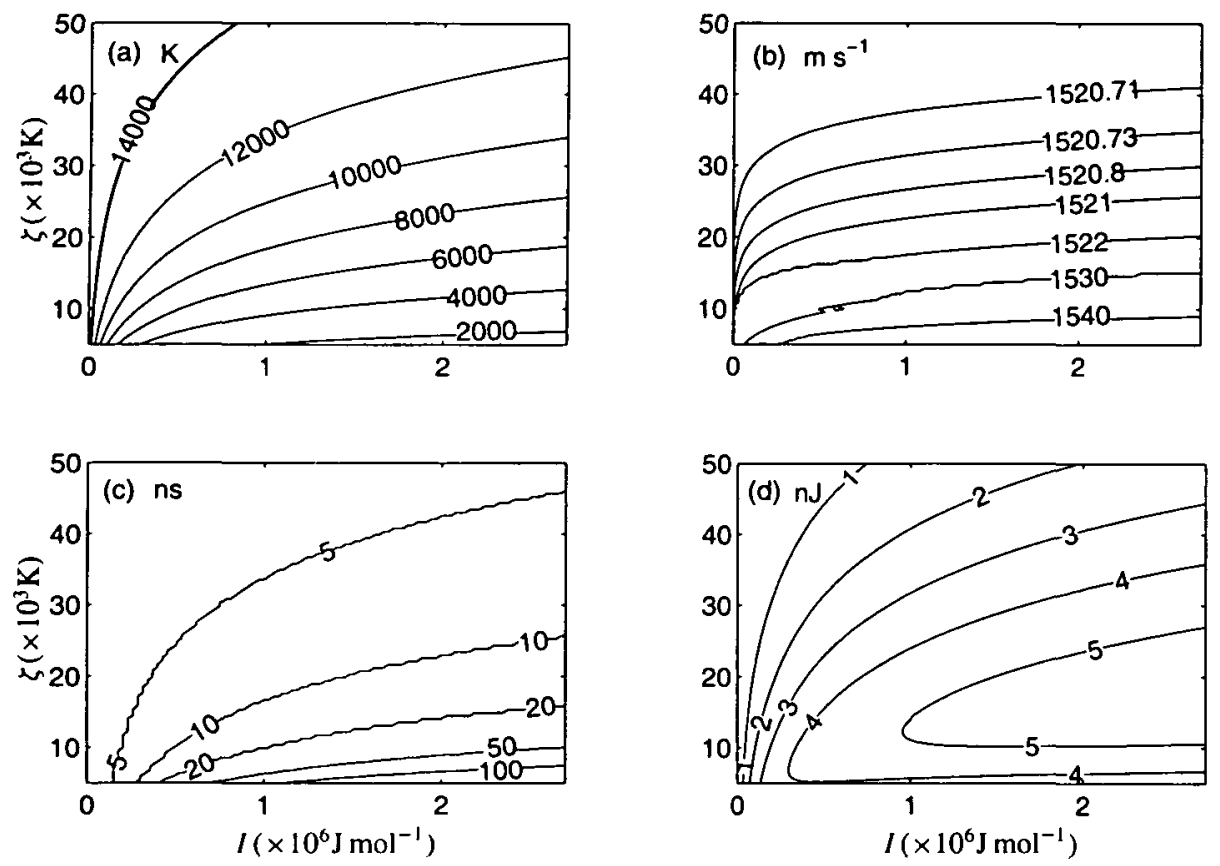

FIGURE 8. Effect of $l$ and $\zeta$ on the collapse characteristics (a) maximum temperature, (b) maximum bubble wall speed, (c) temperature pulse width and (d) total energy of ionisation, of the collapse of a $6 \mu \mathrm{m}$ bubble in the SL regime with $f=25 \mathrm{kHz}, \alpha=1.6$.

The maximum temperatures reached as high as $15000 \mathrm{~K}$. This occurred for very small values of $I$ (ionisation energy). Small values of $\zeta$ (characteristic temperature of ionisation) led to low maximum temperatures, particularly for large $I$. The maxima for infalling wall speed fell within a relatively narrow range. The largest value was $\sim 1540 \mathrm{~ms}^{-1}$, for small $\zeta$. It fell to $\sim 1520 \mathrm{~ms}^{-1}$ in the limit of large $\zeta$ (or, equivalently, zero ionisation). These are similar to values from other more comprehensive models. They are all similar to the speed of sound within the bubble at this high density, so shock waves remain a possibility.

The temperature full-width half-maximum (FWHM) pulse lengths, shown in Figure 8 (c), were shorter for lower $\zeta$ and larger $I$.

The energy of ionisation was generally larger for small $\zeta$ and large $l$, but diminished again for very small $\zeta$. This can be seen in Figure 8 (d). This can be thought of as a competition between the larger amount of ionisation at higher temperatures and the self-quenching of the maximum temperature by ionisation itself. 
TABLE 2. Collapse predictions for different models

\begin{tabular}{lllllllll}
\hline Model & $\begin{array}{l}T_{\max } \\
(\mathrm{kK})\end{array}$ & $\begin{array}{l}v_{\max } \\
\left(\mathrm{ms}^{-1}\right)\end{array}$ & $\begin{array}{l}E_{\text {ionis }} \\
(\mathrm{nJ})\end{array}$ & $\begin{array}{l}\iota_{\max } \\
(\%)\end{array}$ & $\begin{array}{l}\mathrm{FWHM}_{T} \\
(\mathrm{~ns})\end{array}$ & $\begin{array}{l}R_{e} \\
(\mu \mathrm{m})\end{array}$ & $\begin{array}{l}f \\
(\mathrm{kHz})\end{array}$ & $\begin{array}{l}\alpha \\
(\mathrm{atm})\end{array}$ \\
\hline Present & 10 & 1520 & $5^{a}$ & 14 & 5 & 5 & 25 & 1.6 \\
Yasui [43] & 10 & 1900 & 1.4 & & 2 & 5 & 20.6 & 1.35 \\
Ho [17] & 60 & 3100 & 0.3 & 20 & 0.5 & 4.7 & 26.5 & 1.35 \\
Xavier [42] & 20 & & 0.5 & 3 & & 4.5 & 26.5 & 1.35 \\
Kwak [21] & 13 & 1440 & & & & 4.5 & 26.5 & 1.35 \\
Hammer [10] & 16 & 1180 & & & & 5.5 & 33.4 & 1.48 \\
Hilgen [14] & 20 & & & 1 & 1.1 & 5 & 20 & 1.3 \\
\hline
\end{tabular}

${ }^{a}$ Includes energy of chemical reactions and ionisation

In Table 2, values (at normal ionisation), of the quantities discussed above were compared to approximate values from more sophisticated published models. All the models chosen for comparison involved full gas hydrodynamical modelling, except for that of Hilgenfeldt et al. [14], which involves a uniform gas bubble interior.

Hammer and Frommhold [10] calculated that the energies consumed in ionisation and chemical reactions were similar. If that was true for the data presented in Table 2, the relative size of $E_{\text {ionis }}$ (which includes chemical reactions) for the present model would be more consistent with $E_{\text {ionis }}$ (ionisation energy only) for the other models. The total ionisation energy $E_{\max }=\iota_{\max } I$ and the temporal pulse-width for this model are possibly over-estimated. Doubling $\zeta$ and halving $I$ was found to reduce $E_{\max }$ to $\sim 1 \mathrm{~nJ}$ and reduce pulse width to $\sim 3 \mathrm{~ns}$. Notwithstanding the simplicity of the present model and the variation between the predictions of all models, the results are in reasonable agreement with the more sophisticated hydrodynamic models.

The accuracy of the results summarised in Table 2 is still not well known. Notwithstanding the simplicity of the present model and the variation between the predictions of all models, the predictions are in reasonable agreement with the more sophisticated hydrodynamic models. Total ionisation energy $E_{\max }=l_{\max } l$ for this model is perhaps too high, and the temporal pulse width is perhaps slightly too long. Doubling $\zeta$ and halving $I$ was found to reduce $E_{\max }$ to $\sim 1 \mathrm{~nJ}$ and reduce pulse width to $\sim 3 \mathrm{~ns}$.

4.3. Resonances We have established that this model produces similar bubble collapse behaviour to several more complicated models. This allows us now to investigate the effects of ionisation parameters on the bubble's resonance structure, a question which has hitherto received little attention.

Response curves and bifurcation diagrams Frequency bifurcation diagrams were created using both normal ionisation and zero ionisation. No visible difference was generally observed between diagrams produced with these two scenarios at a forcing 
of $1.6 \mathrm{~atm}$. This was true for bubbles in the SL radius regime $(\sim 6 \mu \mathrm{m})$ and for bubbles of larger radii $(\sim 20 \mu \mathrm{m})$.

However, there were instances where some resonances were "missed" as frequency was varied. With no ionisation for a $10 \mu \mathrm{m}$ bubble driven at $0.85 \mathrm{~atm}$, the order of resonances encountered whilst running the system from high frequency to low frequency was (essentially) $R_{3.1}, R_{9,2}, R_{5,1}, R_{11,2}$. In the presence of "normal" ionisation, this sequence became $R_{3,1}, R_{4,1}, R_{11,2}$. By skipping $R_{4,1}$, the oscillation amplitude was approximately halved through a $20 \mathrm{kHz}$ frequency range, and a period-2 bifurcation was avoided. Small changes in other parameters may also lead to large differences in behaviour, as is expected in forced oscillator systems. Attention has been drawn to this here to highlight the experimental consequences of such behaviour. It also leads to the question of whether similar changes are brought on by small differences in modelling of ionisation and chemical reactions.

When $\zeta$ was lowered to enhance ionisation, resonance behaviour did change slightly. This can be seen in Figure 9, where a period-doubling cascade occurred as $\zeta$ was increased. Period-doubling bifurcations were seen to result from increasing the driving amplitude, as in Figure 5. The increase in driving promotes stronger collapse; increasing $\zeta$ has a similar effect because it "frees" more internal energy to contribute to collapse temperature.

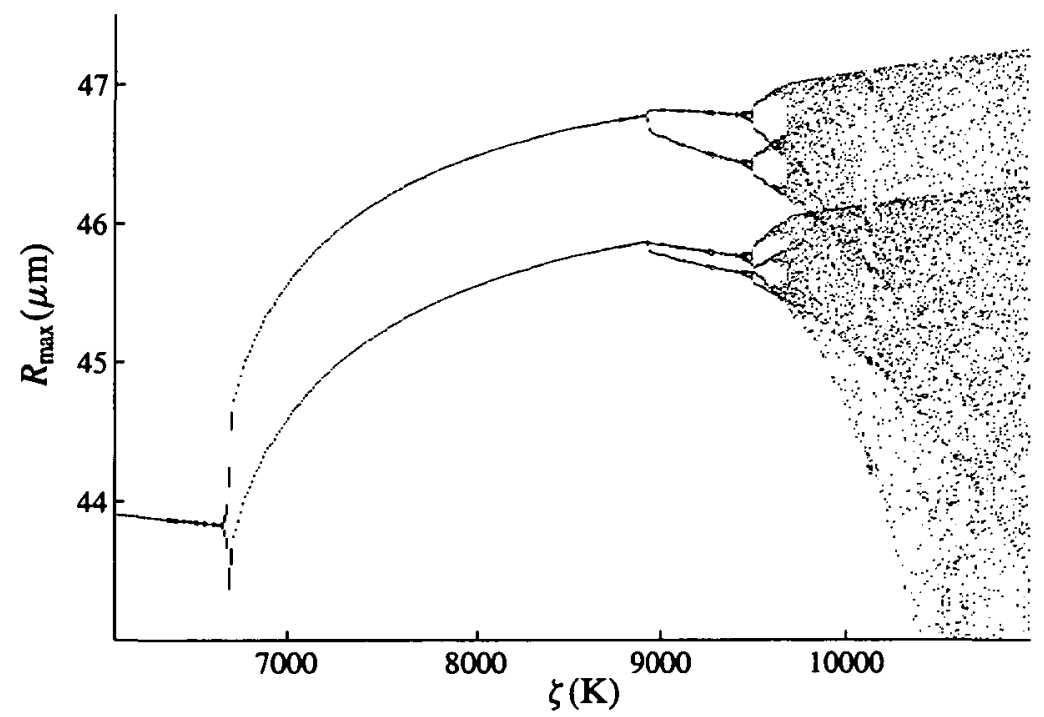

FIGURE 9. Bifurcation diagram in $\zeta: R_{e}=10 \mu \mathrm{m}, f=81.3 \mathrm{kHz}, \alpha=1.3$ and $I=1.35 \times 10^{6} \mathrm{Jmol}^{-1}$.

Resonance maps Figure 10 consists of two resonance maps (which show differentperiod behaviour as different shades) in $\alpha-R_{e}$ space, with $f$ increasing upwards. 
Maps in forcing amplitude $(\alpha)$ and bubble ambient radius $R_{e}$ were used for this investigation, because forcing pressure and initial radius, together with frequency, are the most important control parameters for the oscillating bubble.
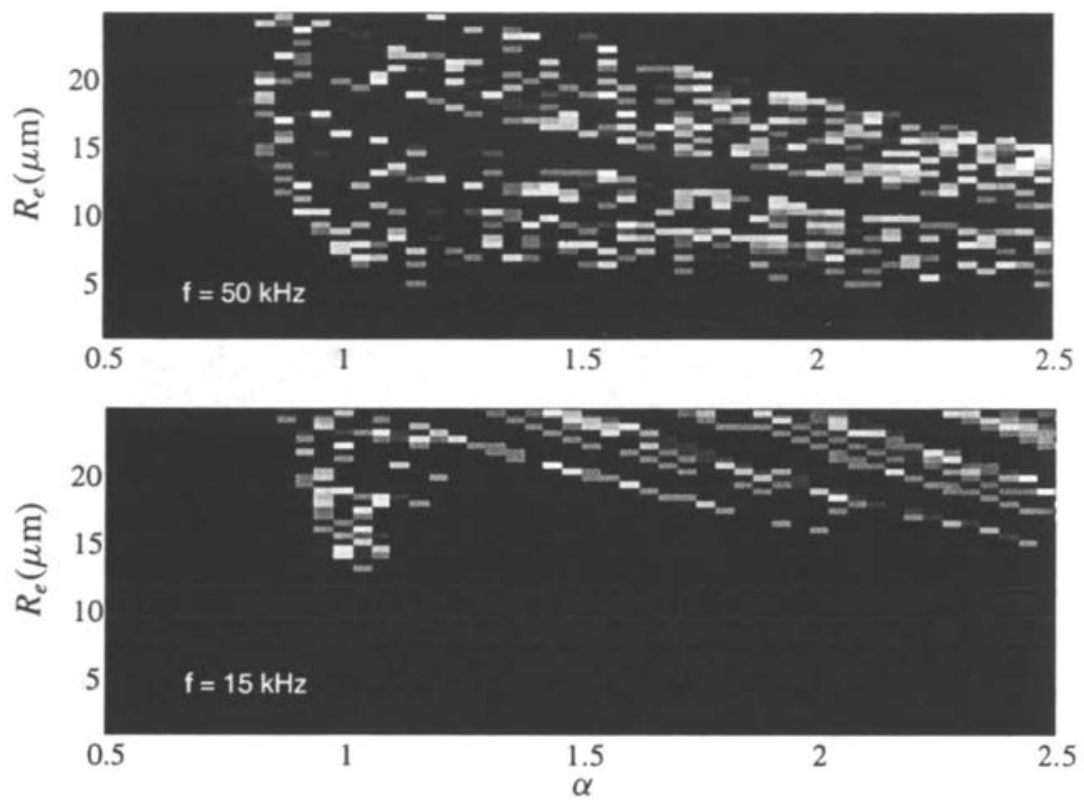

FIGURE 10. Resonance maps at driving frequencies $50 \mathrm{kHz}$ (top) and $15 \mathrm{kHz}$ (bottom), with ionisation $\left(I=6.75 \times 10^{6} \mathrm{Jmol}^{-1} ; \zeta=10000 \mathrm{~K}\right)$. Boxes mark the approximate area of stable SL. The (frequencydependent) $R_{e}$ stability thresholds are based on Hilgenfeldt and Lohse [15].

The structure in Figure 10 comprises higher-period/chaotic islands on a period-1 background, as has been found before at a single frequency [36]. The transition between these behaviours usually occurs via period-doubling. A vertical chaotic band is located consistently at $\alpha \sim 1$ for radii $\gtrsim 10 \mu \mathrm{m}$. The band is eroded at low frequency, because slower driving allows enough time for rebounds to dampen into acquiescence, for a bubble of a given radius [2]. This longer dampening time leads to more stable behaviour.

Resonance horns can be seen protruding from the steady chaotic band toward larger driving amplitudes. The oscillator's softening character causes bending toward smaller radii at larger amplitudes. At the lower frequency, bifurcation depth is generally less than at the higher frequency. Figure 10 shows the onset of bifurcations and chaos at $\sim 6 \mu \mathrm{m}$ for $50 \mathrm{kHz}$ driving and $\sim 13 \mu \mathrm{m}$ for $15 \mathrm{kHz}$. The onset of chaos appears much more abrupt at the higher frequency.

At $f=50 \mathrm{kHz}$ the resonance horns have virtually fused together into two large regions of chaos. Presence or absence (not shown) of the ionisation component had 
little effect at this frequency. The chaotic region is much closer to the SL region at this frequency; however, it is unlikely that collapse temperatures are sufficient to cause SL at $50 \mathrm{kHz}$. Additionally, afterbounce instability is problematic at this frequency [2].

Similar resonance maps of radius versus driving amplitude have been published by Simon [36]; however, no publications were found showing the dependence of structures in these maps on other parameters (in this case, frequency). The maps published here are expected to be of practical interest in experimental situations.

Figure 11 shows fractal structure in a chaotic region of the $\alpha-R_{e}$ space for bubbles of approximately $50 \mu \mathrm{m}$ diameter. Period-1 areas (dark shading) pervade the chaos as a result of stretch-and-fold behaviour.
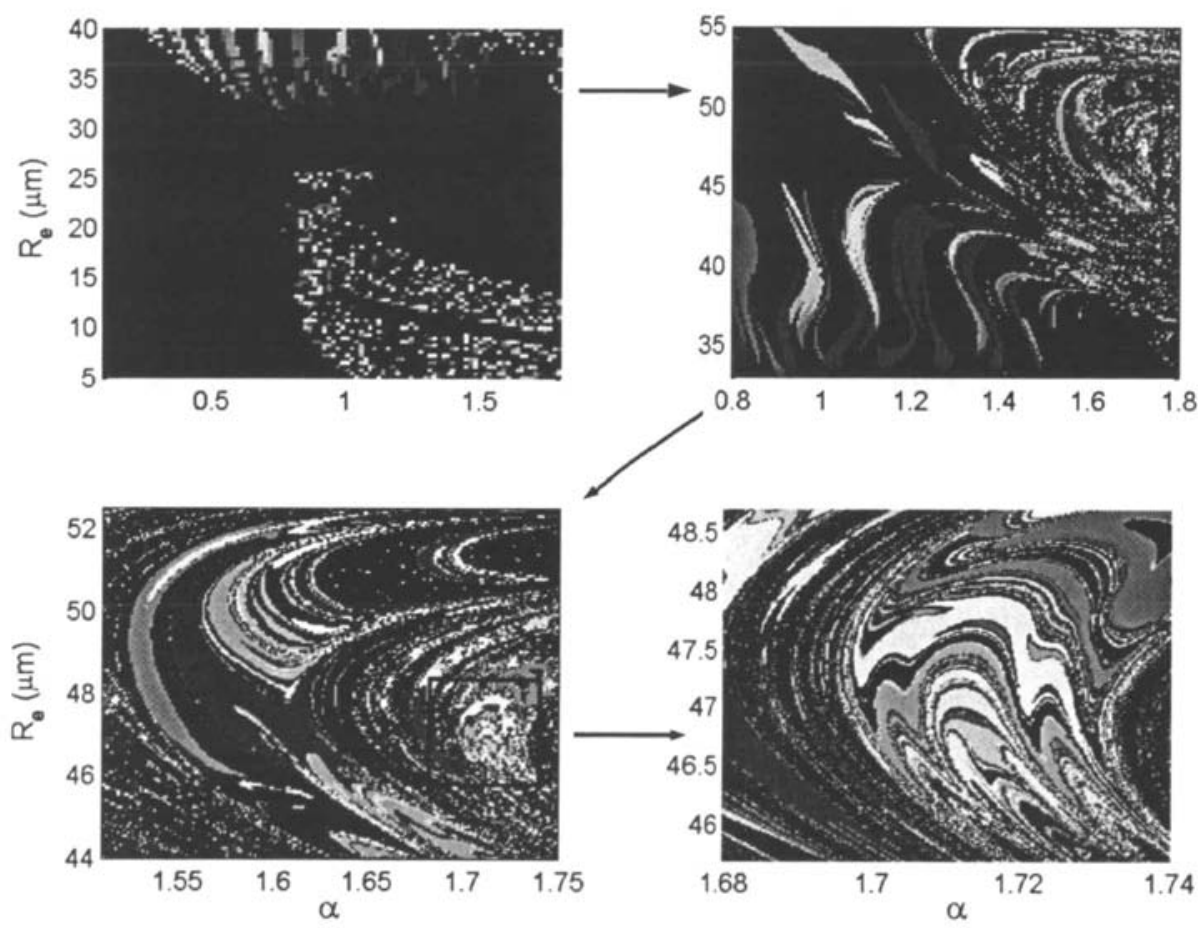

FIGURE 11. Example of strange attractors for bubble resonances in this model.

In most regions of stable, periodic oscillations, changing the ionisation parameters alone did not change the period numbers of resonances. In and close to chaotic regions, however, the resonances became much more sensitive.

Figure 12 shows the dependence of resonance period upon $\zeta$ and $I$, for a bubble driven at a high frequency. Resonances in Figure 12 show a similar dependence on these ionisation parameters as maximum temperatures in Figure 8 (a). This pattern (with considerably more noise) was also found at the lower driving frequencies 


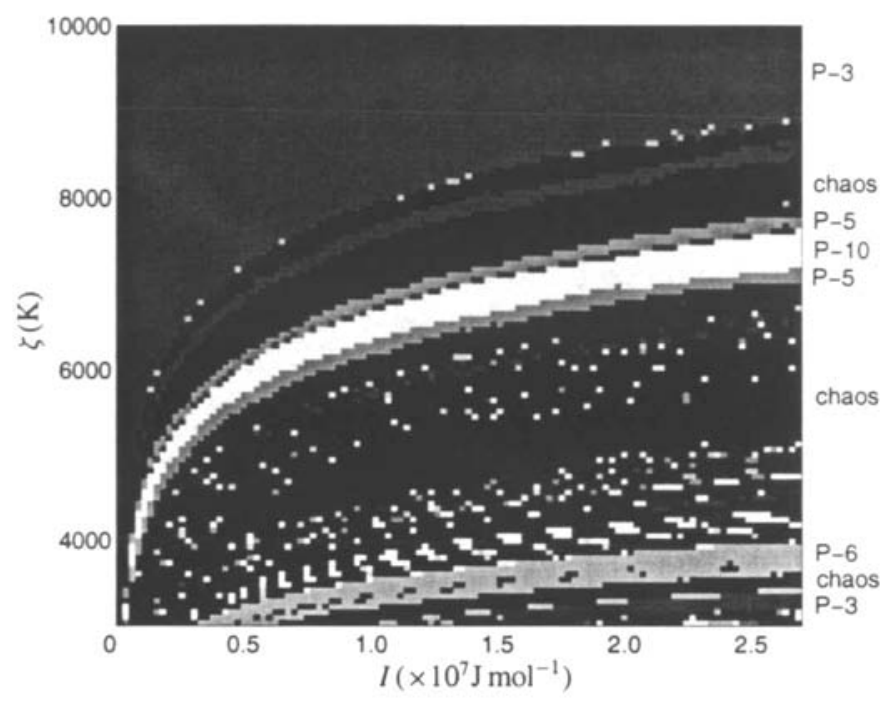

FIGURE 12. Resonance map for a $20 \mu \mathrm{m}$ bubble, forced by $f=170 \mathrm{kHz}$ at $\alpha=1.6$. Resonance periods are indicated at right.

associated with SL.

\section{Summary and Conclusion}

A model of Single-Bubble Sonoluminescence was formulated in which heat transfer and ionisation were included through the first law of thermodynamics. The bubble was assumed to be spherically symmetric, with its gas content at equilibrium.

Equilibria of this stiff system of three first-order ordinary differential equations were found. The linearised system was solved in the case of small- amplitude oscillation to find the system's resonant frequency.

The full nonlinear equations were implemented in MATLAB and solved numerically. Bubble collapse was influenced by changes in the characteristic ionisation temperature $(\zeta)$ more than changes in ionisation energy $(l)$. Maximum collapse temperatures were strongly reduced in the presence of increased ionisation. Other collapse quantities such as collapse duration and maximum bubble-wall velocity were also affected. While the ionisation parameters $(\zeta$ and $I)$ generally had a small effect on the visual appearance of one oscillation cycle, areas in which the effect magnified during many cycles resulted in the resonance period being changed. This sensitivity suggests that it will be difficult to reconcile models and experiments even in areas of stable resonance.

Bifurcations and chaos were observed to emerge for $\sim 6 \mu \mathrm{m}$ bubbles at $50 \mathrm{kHz}$ and 
for $13 \mu \mathrm{m}$ bubbles at $15 \mathrm{kHz}$. This threshold is near to the parameter region where SL has been observed. It remains possible that SL may be observed in a radially chaotic bubble. If this occurs then dynamical models would need to account for ionisation.

The absorption of energy by chemical reactions and ionisation affected dynamics similarly to a slight decrease in forcing amplitude. These high-temperature gas processes reduce the maximum temperature and pressure within the bubble, which are usually most directly controlled by forcing amplitude. The parametrisation of these processes is the main innovation in this model, compared to similar previous models. Because little is known for sure about these processes, continued study of their effects on temperature maxima in collapsing bubbles is warranted.

The effects of this "ionisation" model component are expected to be relevant beyond the (small) SL/ ionisation parameter range, because chemical reaction processes absorb energy in a much larger parameter range. Thus more accurate knowledge of the location of bifurcations and chaos outside the SL region should be of interest in sonochemistry. This model should be of use wherever RP-type dynamics are applicable (subject to various stability requirements), and significant chemical processes affect the energy exchange at collapse.

\section{Appendix A. The Jacobian matrix}

The Jacobian matrix, $J$, was evaluated in the Equilibria and small-amplitude oscillations section using a perturbation technique, in order to study the local behaviour of the system around equilibrium points.

The elements of $J$, denoted $j_{i j}$, are

$$
\begin{aligned}
& j_{11}=0, \quad j_{12}=1, \quad j_{13}=0, \\
& j_{21}=\frac{\phi}{R_{e}^{2}}+\frac{2 \beta}{R_{e}^{3}}-\frac{3 R_{e} T_{e} \xi}{\left(R_{e}^{3}-H^{3}\right)^{2}}-\frac{T_{e} \xi}{R_{e}^{2}\left(R_{e}^{3}-H^{3}\right)}+\frac{7 \kappa_{1} \xi}{R_{e}^{8}}, \\
& j_{22}=\frac{\kappa_{2}}{R_{e}^{7}}-\frac{\lambda}{R_{e}^{2}}-\frac{3 R_{e}^{2} T_{e} \psi}{\left(R_{e}^{3}-H^{3}\right)^{2}}-\frac{4 \pi R_{e}^{2}\left(T_{e} /\left(R_{e}^{3}-H^{3}\right)-\kappa_{1} / R_{e}^{6}\right) \psi}{\left(R_{e}^{3}-H^{3}\right)\left(\mu+\epsilon /\left(e^{\delta / T_{e}} T_{e}^{2}\right)\right)}, \\
& j_{23}=\frac{\xi}{R_{e}\left(R_{e}^{3}-H^{3}\right)}-\frac{4 \pi R_{e}^{2} \chi \psi}{\left(R_{e}^{3}-H^{3}\right)\left(\mu+\epsilon /\left(e^{\delta / T_{e}} T_{e}^{2}\right)\right)}, \\
& j_{31}=0 \\
& j_{32}=\frac{-4 \pi R_{e}^{2}\left(T_{e} /\left(R_{e}^{3}-H^{3}\right)-\kappa_{1} / R_{e}^{6}\right)}{\left(\mu+\epsilon /\left(e^{\delta / T_{e}} T_{e}^{2}\right)\right)} \\
& j_{33}=\frac{-4 \pi R_{e}^{2} \chi}{\left.\left(\mu+\epsilon /\left(e^{\delta / T_{e}}\right) T_{e}^{2}\right)\right)} .
\end{aligned}
$$




\section{References}

[1] C. Adkins, Equilibrium Thermodynamics (Cambridge University Press, Cambridge, 1983).

[2] M. Brenner, S. Hilgenfeldt and D. Lohse, "Single-bubble sonoluminescence", Rev. Modern Phys. 74 (2002) 425-484.

[3] M. Brenner, D. Lohse and T. Dupont, "Bubble shape oscillations and the onset of sonoluminescence", Phys. Rev. Lett. 75 (1995) 954-957.

[4] H. Cheng, M. Chu, P. Leung and L. Yuan, "How important are shock waves to single-bubble sonoluminescence?", Phys. Rev. E 58 (1998) R2705-2708.

[5] L. Forbes, "A series analysis of forced transverse oscillations in a spring-mass system", SIAM J. Appl. Math. 49 (1989) 704-719.

[6] L. Forbes, "An analytical and numerical study of the forced vibration of a spherical cavity", $J$. Sound Vibration 172 (1994) 471-489.

[7] D. Gaitan, L. Crum, C. Church and R. Roy, "Sonoluminescence and bubble dynamics for a single, stable, cavitation bubble", J. Acoust. Soc. Amer. 91 (1992) 3166-3183.

[8] J. Guckenheimer and P. Holmes, Nonlinear oscillations, dynamic systems, and bifurcations of vector fields (Springer, New York, 1983).

[9] D. Hammer and L. Frommhold, "Topical review. Sonoluminescence: how bubbles glow", $J$. Modern Opt. 48 (2001) 239-277.

[10] D. Hammer and L. Frommhold, "Light emission of sonoluminescent bubbles containing a rare gas and water vapor", Phys. Rev. E 65 (2002) 046309-046322.

[11] P. J. Harris, H. J. Al-Awadi and W. K. Soh, "An investigation into the effects of heat transfer on the motion of a spherical bubble", ANZIAM J. 45 (2004) 361-371.

[12] S. Hilgenfeldt, M. Brenner, S. Grossmann and D. Lohse, "Analysis of Rayleigh-Plesset dynamics for sonoluminescing bubbles", J. Fluid Mech. 365 (1998) 171-204.

[13] S. Hilgenfeldt, S. Grossmann and D. Lohse, "A simple explanation of light emission in sonoluminescence", Nature (London) 398 (6726) (1999) 402-405.

[14] S. Hilgenfeldt, S. Grossmann and D. Lohse, "Sonoluminescence light emission", Phys. Fluids 11 (1999) 1318-1330.

[15] S. Hilgenfeldt and D. Lohse, "Predictions for upscaling sonoluminescence", Phys. Rev. Lett. 82 (1999) 1036-1039.

[16] S. Hilgenfeldt, D. Lohse and M. Brenner, "Phase diagrams for sonoluminescing bubbles", Phys. Fluids 8 (1996) 2808-2826.

[17] C. Ho, L. Yuan, M. Chu, P. Leung and W. Wei, "Effects of ionization in single-bubble sonoluminescence", Phys. Rev. E 65 (2002) 041201-1-041201-12.

[18] J. B. Keller and I. I. Kolodner, "Damping of underwater bubble explosion oscillations", J. Appl. Phys. 27 (1956) 1152-1161.

[19] J. Ketterling and R. Apfel, "Shape and extinction thresholds in sonoluminescence parameter space", J. Acoust. Soc. Amer. 107 (2000) L13-L18.

[20] L. Kondic, J. Gersten and C. Yuan, "Theoretical studies of sonoluminescence radiation: radiative transfer and parametric dependence", Phys. Rev. E 52 (1995) 4976-4990.

[21] H. Kwak, J. Lee and S. Karng, "Bubble dynamics for single-bubble sonoluminescence", J. Phys. Soc. Japan 70 (2001) 2909-2917.

[22] W. Lauterborn, "Numerical investigation of nonlinear oscillations of gas bubbles in liquids", $J$. Acoust. Soc. Amer. 59 (1976) 283-293.

[23] W. Lauterborn and R. Mettin, "Response curves of bubbles", in Proceedings of the $16 \mathrm{~h} / \mathrm{CA} / \mathrm{l} 35 \mathrm{th}$ ASA Meeting Seatle, WA. USA, (Acoustial Society of America (ASA), 1998), pp. 2281-2282.

[24] W. Lauterborn and U. Parlitz, "Methods of chaos physics and their application to acoustics", J. Acoust. Soc. Amer. 84 (1988) 1975-1993. 
[25] M. T. Levinsen, N. Weppenaar, J. S. Dam, G. Simon and M. Skogstad, "Direct observation of period-doubled nonspherical states in single-bubble sonoluminescence", Phys. Rev. E 68 (2003) 35303.

[26] D. Lide (ed.), CRC Handbook of Chemistry and Physics (CRC Press, Boca Ranton, 1995).

[27] H. Lin, B. Storey and A. Szeri, "Inertially driven inhomogeneities in violently collapsing bubbles: the validity of the Rayleigh-Plesset equation", J. Fluid Mech. 452 (2002) 145-162.

[28] R. Lofstedt, S. Weninger, S. Putterman and B. Barber, "Sonoluminescing air bubbles rectify argon", Phys. Rev. E 51 (1995) 4400-4410.

[29] W. Moss, D. Young, J. Harte, J. Levatin, B. Rozsnyai, G. Zimmerman and I. Zimmerman, "Computed optical emissions from a sonoluminescing bubble", Phys. Rev. E 59 (1999) 2986-2992.

[30] U. Parlitz, "Common dynamical features of periodically driven strictly dissipative oscillators", Internat. J. Bifurcation Chaos 3 (1993) 703-715.

[31] U. Parlitz, C. Englisch, C. Scheffczyk and W. Lauterborn, "Bifurcation structure of bubble oscillators", J. Acoust. Soc. Amer. 88 (1990) 1061-1077.

[32] U. Parlitz and W. Lauterborn, "Resonances and torsion numbers of driven dissipative nonlinear oscillators”, Z. Naturforsch 41 (a) (1985) 605-614.

[33] M. Plesset, "The dynamics of cavitation bubbles", J. Appl. Mech. 16 (1949) 277-282.

[34] L. Rayleigh, "On the pressure developed in a liquid during the collapse of a spherical cavity", Phil. Mag. 34 (1917) 94-98.

[35] C. Scheffczyk, U. Parlitz, T. Kurz, W. Knop and W. Lauterborn, "Comparison of bifurcation structures of driven dissipative nonlinear oscillators", Phys. Rev. A 43 (1991) 6495-6502.

[36] G. Simon, P. Cvitanovic, M. Levinsen, I. Csabai and A. Horvath, "Periodic orbit theory applied to a chaotically oscillating gas bubble in water", Nonlinearity 15 (2002) 25-43.

[37] B. Storey and A. Szeri, "Mixture segregation within sonoluminescing bubbles", J. Fluid Mech. 396 (1999) 203-221.

[38] S. Umemura, N. Yumita and R. Nishigaki, "Enhancement of ultrasonically induced cell damage by a gallium-porphyrin complex, ATX-70", Japan. J. Cancer Res. 84 (1993) 582-588.

[39] E. Unger, T. Matsunaga, T. McCreery, P. Schumann, R. Sweitzer and R. Quigley, "Therapeutic applications of microbubbles", Eur. J. Radiology 42 (2002) 160-168.

[40] G. Vazquez and S. Putterman, "Temperature and pressure dependence of sonoluminescence", Phys. Rev. Lett. 85 (2000) 3037-3040.

[4I] V. Vuong and A. Szeri, "Sonoluminescence and diffusive transport", Phys. Fluids 8 (1996) 23542364.

[42] C. Xavier and R. Clemente. "Dissociation and ionization in sonoluminescence", J. Phys. Soc. Japan 70 (2001) 387-393.

[43] K. Yasui, "Alternative model of single-bubble sonoluminescence", Phys. Rev. E 56 (1997) 67506760. 